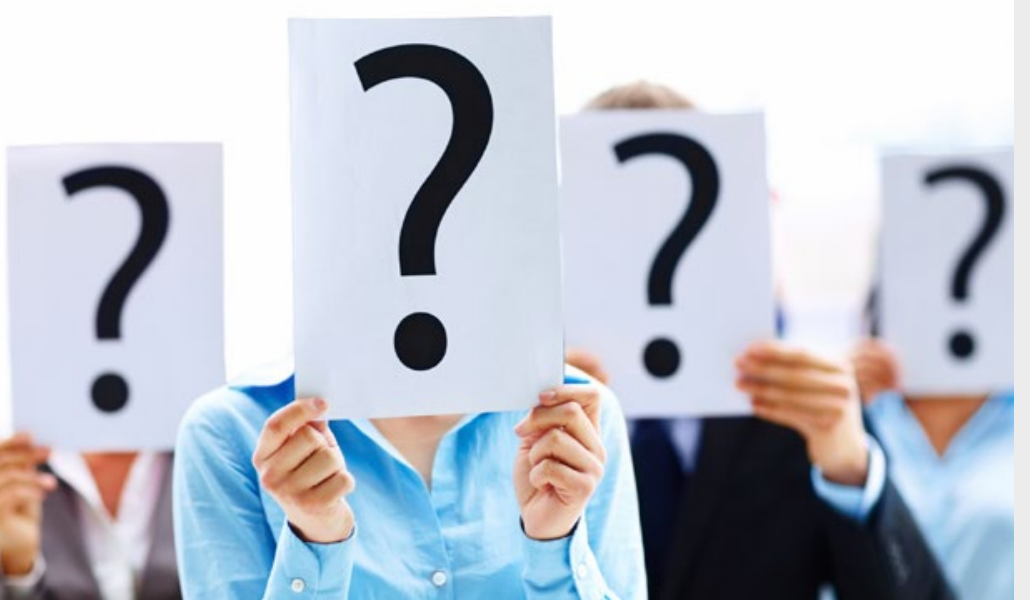

Der Krebsinformationsdienst (KID) des Deutschen Krebsforschungszentrums greift in Fachpublikationen, Presseund Informationsdiensten verarbeitete Ergebnisse und Entwicklungen in onkologischer Forschung, Klinik und Versorgung auf. Für eine orientierende Einordnung werden wissenschaftlicher Hintergrund und Stellenwert beleuchtet. Die kurze Darstellung der Sachverhalte, ihrer praktischen Relevanz und der zugrunde liegenden Forschungsergebnisse kann auch Ihnen bei der Beantwortung von Patientenfragen helfen.

\title{
Was kann Vibration Response Imaging?
}

\begin{abstract}
Hintergrund
Vibration Response Imaging (VRI) ist eine von einem kleinen israelischen Unternehmen entwickelte nichtinvasive diagnostische Methode zur Lungenfunktionsprüfung (Deep Breeze ${ }^{\mathrm{Tm}}$ ). Sie basiert auf der Messung des Luftstroms in den Bronchien: Während 3 bis 5 Atemzyklen erfassen 40 auf dem Rücken des Patienten angebrachte Sensoren - 20 über jedem Lungenflügel - die Energie der Luftvibration in den Atemwegen. Jede Lunge wird dabei wie bei der Ventilations-/ Perfusionsszintigraphie, dem aktuellen Goldstandard, in 3 getrennt beurteilte Sektoren unterteilt. Ergebnis der Auswertung ist ein Bild der Lungen, das die Vibrationsenergie in den Sektoren in Graustufen umsetzt [1]. In der Onkologie ist die Prädiktion der postoperativen Lungenfunktion vor einer Tumorresektion eine wichtige Untersuchung. Hier hat VRI potenziell mehrere Vorteile: Die Untersuchung ist einfach und ohne großen apparativen Aufwand in etwa 4-6 min durchzuführen; die Auswertung mit einer speziellen Software dauert etwa $5 \mathrm{~min}$, sodass die Ergebnisse schnell vorliegen. Eine Strahlenbelastung ist nicht gegeben. In Studien wurde VRI u. a. bei Patienten mit potenziell resektablen Tumoren mit den konventionellen Methoden der Lungenfunktionsprüfung verglichen mit der Frage, ob sich damit eine einfachere und kostengünstige Alternative bieten könnte. Dass es sich beim VRI auch um ein im weiteren Sinne bildgebendes Verfahren handelt, kann bei Pa-
\end{abstract}

tienten zu Missverständnissen hinsichtlich der Nutzbarkeit für Früherkennung und Diagnose von Lungentumoren führen. Aber morphologische Informationen liefert das VRI nicht.

\section{Stellenwert in der Lungenfunktionsdiagnostik weiter fraglich}

VRI ist nach wie vor ein experimentelles Verfahren ohne gesicherten Stellenwert. Die Methode hatte in den wenigen aktuelleren Studien bei onkologischer Indikation eine ähnliche Vorhersagekraft wie Spirometrie und Ventilations-/Perfusionsszintigraphie bzw. Ganzkörper-Plethysmographie mit einer hohen Korrelation von vorausgesagter und tatsächlicher postoperativen Funktionskapazität und guter Übereinstimmung mit der Szintigraphie - bei allerdings großen Konfidenzintervallen [1,2]. Aufgrund der beobachteten Variabilität der Prädiktion bleibt offen, ob die Methode eine echte Alternative zur Ventilations-/Perfusionsszintigraphie darstellen könnte. Für die Früherkennung oder Diagnostik von Lungentumoren ist sie nicht geeignet.

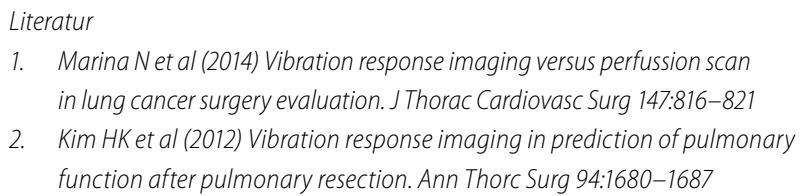

\section{Kann Ingwer die Wirkung von Chemotherapie beeinträchtigen?}

\section{Hintergrund}

Ingwer gilt als ,rundum gesund “ und hat auch hierzulande zunehmend Eingang in die Ernährung gefunden, nicht nur als Nahrungsergänzungsmittel. Zudem gibt es (wenn auch noch nicht mit hoher Evidenz belegte) Hinweise, dass Ingwer sowohl die Symptome von Reisekrankheit wie auch chemotherapiebedingter Übelkeit und Erbrechen lindern kann [1]. Die Frage, ob pharmakologisch aktive Inhaltsstoffe auch zu Interaktionen mit in der Krebstherapie eingesetzten Medikamenten führen, ist berechtigt. Bekannte und möglicherweise klinisch relevante Wechselwirkungen von Ingwer ergeben sich bei antikoagulati- ven und antiphlogistischen Therapien: Durch Hemmung der Thromboxanbildung kann das Blutungsrisiko erhöht werden. Perioperativ und generell bei Patienten mit Gerinnungsstörungen sollen Ingwerpräparate nicht angewendet werden.

Da Ingwer den Blutzuckerspiegel senkt, wird die Wirkung antidiabetischer Therapien verstärkt. Der Plasmaspiegel von Tacrolimus wird erhöht, der von Cyclosporin im Tiermodell gesenkt [2]. Interaktionen mit Zytostatika oder anderen Krebsmedikamenten sind dagegen bisher nicht dokumentiert. In-vitro-Tests deuten zwar darauf hin, dass Inhaltsstoffe von Ingwer möglicherweise die Aktivität und Expression diverser Zyto- 